\footnotetext{
${ }^{7}$ The valence band in our model contains 36 more orbitals than the conduction band due to "surface states" (or "dangling" orbitals on the surface atoms; see Fig. 1). In our model the energies of the se orbitals are in the valence band and, as such, penetrate well into the bulk. Since it is not possible therefore to distinguish them, the total energy is determined by filling all orbitals in the valence band. This must be done in order to fill properly all of the bulk orbitals. In doing this, surface orbitals are also being filled, but we argue that they should not contribute significantly to the elastic forces.

${ }^{8}$ H. J. McSkimin and W. L. Bond, Phys. Rev. 105, 116 (1957). A recent measurement gives $C_{12}=2.75$ [Markham, unpublished, and reported in Physical Properties of Diamond, edited by R. Berman (Oxford Univ., London, 1965), p. 415] giving even better agreement.

${ }^{9}$ W. V. Smith, P. P. Sorokin, I. L. Gelles, and G. J.
}

Lasher, Phys. Rev. 115, 1546 (1959).

${ }^{10}$ P. J. Dean, Phys. Rev. 139, A588 (1965).

${ }^{11}$ P. Denham, E. C. Lightowlers, and P. J. Dean, Phys. Rev. 161, 762 (1967).

${ }^{12}$ A. B. Lidiard and A. M. Stoneham, in Science and Technology of Industrial Diamonds, edited by J. Burls (Eyre and Spottiswoode, London, 1967), Vol. I, p. 1.

${ }^{13}$ G. D. Watkins, in Radiation Effects in Semiconductors, edited by F. Vook (Plenum, New York, 1968), p. 67.

${ }^{14}$ R. J. Cook and D. H. Whiffen, Proc. Roy. Soc., Ser. A 295, 99 (1966).

${ }^{15}$ The details of these estimates will be included in a more complete account to be published later.

${ }^{16}$ The $2 s$ character of the MO appears somewhat too low to account for the isotropic hyperfine interaction, particularly for the nitrogen atom. The difficulty here may be our limited basis set-particularly ignoring the $1 s$ core.

\title{
CORE TRANSITIONS AND DENSITY OF CONDUCTION STATES IN THE III-V SEMICONDUCTORS
}

\author{
M. Cardona, * W. Gudat, $\dagger$ E. E. Koch, $\ddagger$ M. Skibowski, $\ddagger$ B. Sonntag, $\dagger$ and P. Y. Yu§ \\ Deutsches Elektronen-Synchrotron DESY, Hamburg, Germany
}

(Received 3 August 1970)

\begin{abstract}
The absorption and reflection spectra of several amorphous and crystalline III- $\mathrm{V}$ semiconductors have been measured in the region from 10 to $30 \mathrm{eV}$ where transitions from the outermost $d$ levels of the cation cores occur. The use of the continuous spectrum of synchrotron radiation of DESY enabled us to resolve considerable structure in the crystalline samples. This structure can be interpreted as due to structure in the density of conduction states and to the spin-orbit splitting of the core level.
\end{abstract}

The optical transitions from the valence to the conduction bands of crystalline III-V compounds show a great deal of structure in the energy region below $7 \mathrm{eV}$. This structure is now quite well understood and has been responsible for the recent advances in our knowledge of the energy bands of these materials. ${ }^{1}$ For absorption and reflection spectroscopy above $7 \mathrm{eV}$, gas-discharge sources with relatively widely spaced lines, which are conventionally used, ${ }^{2,3}$ limit the resolution rather drastically. This fact is particularly critical in the region where the first core transitions of the III-V compounds $(\sim 20 \mathrm{eV})^{3}$ occur: The hot-cathode argon lamps used have typical separations of about $1 \mathrm{eV}$ between adjacent lines, equal to the expected spinorbit splitting of the core levels ${ }^{4}(0.53 \mathrm{eV}$ for $\mathrm{Ga}$, $0.97 \mathrm{eV}$ for $\mathrm{In}$ ) and to the separation between peaks in the density of conduction states. ${ }^{5}$ For this reason we measured the absorption and reflection spectra of the crystalline and the amorphous modifications of GaP, GaAs, GaSb, InP, InAs, and $\mathrm{InSb}$ in the $10-30 \mathrm{eV}$ region using as a source the continuous synchrotron radiation of DESY. 6,7 We found, in all cases, considerable structure which can be assigned to structure in the density of conduction states. ${ }^{5}$ All the In compounds measured also showed structure which could be correlated with the splitting of the $d$ levels of In. This splitting is too small to be observed in most of the Ga compounds.

The samples for reflection measurements were mechanically polished and etched single crystals with flash-evaporated layers; vacuum-deposited layers were used in transmission work. These layers were prepared as either amorphous or crystalline by varying the substrate temperature. The transmission samples were deposited on a $\mathrm{KCl}$-coated microscope slide. They were then floated on water and picked up with a copper mesh. The transmission spectra of crystalline films and the reflection spectra from etched single crystals as well as from crystalline layers showed essentially the same structure. The amorphous layers gave only broad structure similar to that obtained with polished but un- 


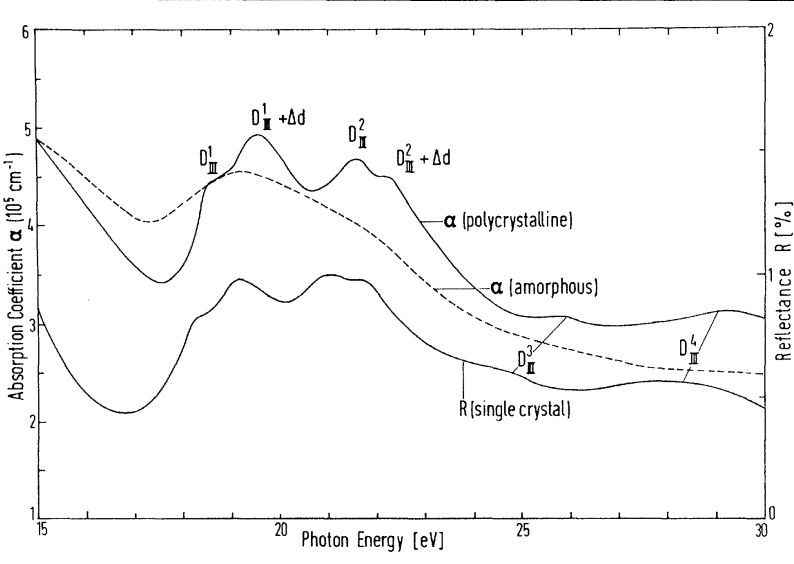

FIG. 1. Absorption spectra of crystalline (substrate temperature $T_{s}=300^{\circ} \mathrm{C}$ ) and amorphous $\left(T_{s}=25^{\circ} \mathrm{C}\right)$ InAs and reflection spectrum of single-crystal InAs etched in methanol with iodine. The values of the absorption coefficient are good to $\pm 20 \%$, the absolute reflectance values to $\pm 50 \%$.

etched single crystals. Two normal-incidence monochromators operating in a modified Wads worth mounting ${ }^{8}$ were used with 1440 and 2400 line $/ \mathrm{mm}$ gratings. The width of the observed structure was in no case determined by instrumental resolution (2 ̊̊ over the whole energy range). The detector was a Bendix M 306 open magnetic multiplier.

Figure 1 shows the absorption spectrum of amorphous and crystalline InAs films and the reflection spectrum of a polished and etched single crystal of the same material. The amorphous material shows only broad structure, in the region of the $d$ electron transitions of In. Considerable fine structure is apparent, however, both in the reflection and in the transmission spectrum of the crystalline materials. This structure consists of four main peaks $\left(D_{\mathrm{III}}{ }^{1}, D_{\mathrm{III}}{ }^{1}+\Delta_{d}\right.$, $\left.D_{\mathrm{III}}{ }^{2}, D_{\mathrm{III}}{ }^{2}+\Delta_{d}\right)$ and two additional broad maxima $\left(D_{\mathrm{II}}{ }^{3}, D_{\mathrm{III}}{ }^{4}\right)$ at higher energies. Between 11 and 16 $\mathrm{eV}$ the reflection and absorption decrease monotonically by a factor of 10 . This behavior is determined by the plasma energy of the valenceband electrons. ${ }^{3,9}$ The energies of the peaks observed in reflection and in absorption for this and other crystalline III-V compounds are listed in Table I. The maxima recently observed in electron energy-loss spectra are included. ${ }^{9}$ Good agreement exists between the positions of the reflection and absorption peaks, although shifts smaller than the peak linewidth $(\sim 1 \mathrm{eV})$ occur; in general the reflection peaks of Table I lie about a few tenths of an $\mathrm{eV}$ below the corresponding absorption peaks. It is easy to notice that
Table I. Energies of the peaks observed in the III-V compounds, related to transitions from the outermost $d$ levels of the cation core to the conduction band.

\begin{tabular}{ccccccc}
\hline & GaP & GaAs & GaSb & InP & InAs & InSb \\
\hline$D_{\text {III }}{ }^{\mathrm{c}}$ & $20.6^{\mathrm{a}}$ & $20.9^{\mathrm{a}}$ & $20.8^{\mathrm{a}}$ & $18.8^{\mathrm{a}}$ & $18.5^{\mathrm{a}}$ & $18.3^{\mathrm{a}}$ \\
& $20.9^{\mathrm{b}}$ & $20.6^{\mathrm{b}}$ & $20.4^{\mathrm{b}}$ & $18.8^{\mathrm{b}}$ & $18.5^{\mathrm{b}}$ & \\
& $21.3^{\mathrm{c}}$ & $21.0^{\mathrm{c}}$ & $20.8^{\mathrm{c}}$ & & & \\
$D_{\text {III }}{ }^{1}+\Delta_{d}$ & $21.2^{\mathrm{a}}$ & & & $19.75^{\mathrm{a}}$ & $19.5^{\mathrm{a}}$ & $19.2^{\mathrm{a}}$ \\
& & & & $19.75^{\mathrm{b}}$ & $19.4^{\mathrm{b}}$ & $18.9^{\mathrm{b}}$ \\
$D_{\text {III }}{ }^{2}$ & $23.1^{\mathrm{a}}$ & $23.0^{\mathrm{a}}$ & $22.4^{\mathrm{a}}$ & $21.6^{\mathrm{a}}$ & $21.55^{\mathrm{a}}$ & $20.8^{\mathrm{a}}$ \\
& $23.2^{\mathrm{b}}$ & $22.9^{\mathrm{b}}$ & $22.2^{\mathrm{b}}$ & $21.6^{\mathrm{b}}$ & $21.3^{\mathrm{b}}$ & $20.9^{\mathrm{b}}$ \\
& $23.3^{\mathrm{c}}$ & $23.2^{\mathrm{c}}$ & $22.5^{\mathrm{c}}$ & & $21.6^{\mathrm{c}}$ & $21.0^{\mathrm{c}}$ \\
$D_{\text {III }}{ }^{2}+\Delta_{d}$ & & & & $22.4^{\mathrm{a}}$ & $22.3^{\mathrm{a}}$ & $21.5^{\mathrm{a}}$ \\
& & & & $22.5^{\mathrm{b}}$ & $22.1^{\mathrm{b}}$ & \\
$D_{\text {III }}{ }^{3}$ & & & & & $26.0^{\mathrm{a}}$ & \\
& & & & & & $25^{\mathrm{c}}$ \\
& $25^{\mathrm{b}}$ & & $24.4^{\mathrm{b}}$ & $24.4^{\mathrm{b}}$ & $24.7^{\mathrm{b}}$ & \\
$D_{\text {III }}{ }^{4}$ & & & $28.4^{\mathrm{a}}$ & $28.5^{\mathrm{a}}$ & $29.2^{\mathrm{a}}$ & \\
& & & $28.3^{\mathrm{b}}$ & $29.8^{\mathrm{b}}$ & $27.5^{\mathrm{b}}$ & \\
& & & $28.5^{\mathrm{c}}$ & & $27.5^{\mathrm{c}}$ & \\
\hline \hline
\end{tabular}

${ }^{\mathrm{a}}$ Transmission of thin polycrystalline films.

${ }^{b}$ Reflection of single crystals.

${ }^{\mathrm{c}}$ Electron energy loss; see Ref. 9 .

the $D_{\mathrm{III}}{ }^{1}-\left(D_{\mathrm{II}}{ }^{1}+\Delta_{d}\right)$ and the ${D_{\mathrm{III}}}^{2}-\left(D_{\mathrm{II}}{ }^{2}+\Delta_{d}\right)$ splittings do not appear in the Ga compounds [with the exception of $D_{\mathrm{III}}{ }^{1}-\left(D_{\mathrm{II}}{ }^{1}+\Delta_{d}\right)$ in GaP]. This fact suggests that these splittings are related to the spinorbit splitting of the $d$ core level of the metallic atom: $0.97 \mathrm{eV}$ for In and $0.53 \mathrm{eV}$ for $\mathrm{Ga}$ (too small to be resolved). ${ }^{4}$ The remaining $D_{\mathrm{mI}}{ }^{1}-D_{\mathrm{mI}}{ }^{2}$ splitting must be related to the density of conduction states since the $d$ bands are essentially flat, or to an energy dependence of the transition matrix element. Matrix-element structure sharper than $1 \mathrm{eV}$ is rather unlikely, as follows from our experience with the valence-band transitions of these materials and from the computation of energy dependence of some core matrix elements by Klima. ${ }^{9}$ Also, the reasonable agreement of the observed structure with structure in the density of conduction states, as discussed below, suggests that structure in the matrix element does not play an important role.

Figure 2 shows the excess absorption due to the $d$ transitions for the crystalline material, obtained by extrapolation of the tail of the valence-band transitions from $\omega<17$ to $\omega>17 \mathrm{eV}$. Such extrapolation was made with the law $\alpha$ $\propto \omega^{-2.4}$, which fits the observed absorption for $\omega<17 \mathrm{eV}$. The dashed curve is the absorption spectrum calculated on the basis of the computed density of conduction states, taking into account the spin-orbit splitting of the $d$ core levels of In 


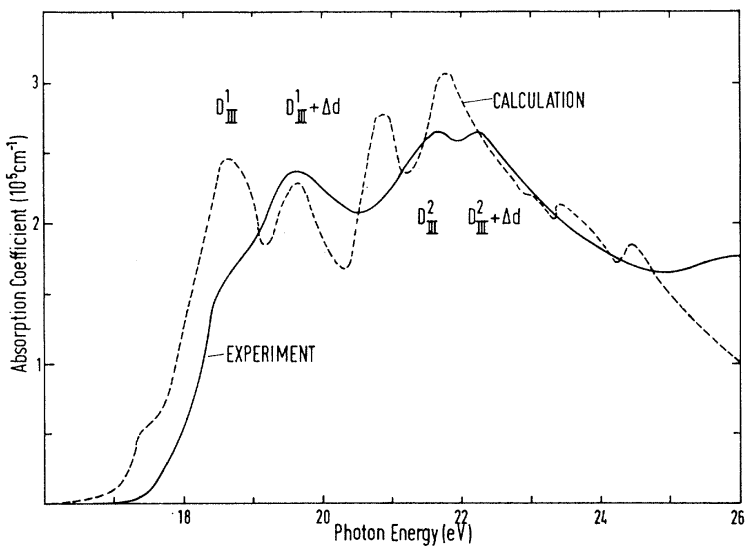

FIG. 2. Contribution of the $D_{I I I}$ transitions to the absorption spectrum of Fig. 1 and absorption calculated with Eq. (1) and the density of states of Ref. 5. The energy $\omega_{d}$ of Eq. (1) has been determined by fitting the observed energy of the $D_{\text {III }}^{1}$ peak.

and the appropriate degeneracy factors. We used the expression (in atomic units)

$$
\begin{aligned}
\alpha \simeq \frac{\omega \epsilon_{2}}{c} \frac{4 \pi^{2}}{3 c \omega} P_{d}{ }^{2}\left[3 N_{d}\left(\omega-\omega_{d}\right)\right. & \\
& \left.+2 N_{d}\left(\omega-\omega_{d}-\Delta_{d}\right)\right],
\end{aligned}
$$

where the real part of the refractive index $n$ has been taken equal to $1 .^{3} N_{d}$ is the density of conduction states, $\omega$ the photon energy, $\omega_{d}$ the energy difference between the bottom of the conduction band and the core level, and $P_{d}$ the dipole matrix element. The agreement of the gross features of the experimental line shape with the calculated one is quite satisfactory. Fine structure, very similar to that observed, is also present in the computed curve. The splitting between the two lower-energy computed lines is indeed of spin-orbit origin and also that between of the third and fourth line, although a slight deformation due to a number of peaks in the density of conduction states seems to be present. The $D_{\text {III }}{ }^{1-}$ $D_{\text {III }}{ }^{2}$ splitting is due to the main features in the density of conduction states. It corresponds essentially to the splitting between the two lowest conduction bands: The lowest conduction band has a very flat region in the vicinity of the $X$ point, while the second lowest band has a similar region near the $L$ point. ${ }^{5}$ These regions give rise to the $D_{\mathrm{II}}{ }^{1}-D_{\mathrm{II}}{ }^{2}$ splitting. The value of the average matrix element $P_{d}$ required in Eq. (1) for the curve of Fig. 2 is 0.14 a.u., a very rea- sonable value in view of the fact that the typical matrix element for valence-band transitions is 0.6 . This value is also reasonable when compared with those calculated for core transitions in germanium and silicon ${ }^{10}$ and with that determined experimentally for the $d$ transitions of germanium $\left(P_{d} \simeq 0.13\right) .{ }^{11}$

Figure 1 shows two additional peaks, $D_{\mathrm{II}}{ }^{3}$ and $D_{\text {III }}{ }^{4}$; these weak peaks have been systematically observed for a number of III-V compounds, as shown in Table I. While their origin is not clear, they are likely to be related, especially $D_{\mathrm{III}}{ }^{4}$, to higher conduction bands not included in the density of states of Fig. 2 and Ref. 5. A look at the calculated band structure of InAs shows a rather flat conduction band located about $10 \mathrm{eV}$ above the bottom of the lowest conduction band and with $\Gamma_{15}$ ( $p$ - and $d$-like) symmetry at $k=0$. This band may be responsible for the $D_{\text {III }}{ }^{4}$ peaks.

*J. S. Guggenheim Foundation Fellow, on leave from Brown University, Providence, R. I.

$\dagger$ II. Institut für Experimentalphysik der Universität Hamburg, Hamburg, Germany.

\$S Sektion Physik der Universität München, München, Germany.

§General Telephone and Electronics Fellow, on leave from Brown University, Providence, R. I.

${ }^{1} \mathrm{~J}$. C. Phillips, in Solid State Physics, edited by H. Ehrenreich, F. Seitz, and D. Turnbull (Academic, New York, 1966), Vol. 18, p.1; M. Cardona, ibid., Suppl. 11.

${ }^{2}$ J. A. R. Samson, Techniques of Vacuum Ultraviolet Spectroscopy (Wiley, New York, 1967).

${ }^{3}$ H. R. Philipp and H. Ehrenreich, Phys. Rev. Lett. 8, 92 (1962), and Phys. Rev. 129, 1550 (1963).

${ }^{4} \mathrm{~F}$. Herman and S. Skillman, Atomic Structure calculations (Prentice-Hall, Englewood Cliffs, N. J., 1963).

${ }^{5}$ C. W. Higginbotham, F. H. Pollak, and M. Cardona, in Proceedings of the International Conference on the Physics of Semiconductors, Moscow, U.S.S.R., 1968 (Nauka, Leningrad, U.S.S.R., 1968), Vol. 1, p. 57.

${ }^{6}$ R. Haensel and C. Kunz, Z. Angew. Phys. 23, 276 (1967).

${ }^{7}$ R. P. Godwin, in Springer Tracts in Modern Physics, edited by G. Höhler (Springer, New York, 1969), Vol. 51.

${ }^{8} \mathrm{M}$. Skibowski and W. Steinmann, J. Opt. Soc. Amer. 57, 112 (1967)。

${ }^{9}$ C. v. Festenberg, Z. Phys. 227, 453 (1969).

${ }^{10} \mathrm{~J}$. Klima, J. Phys. C: Solid State Phys。 3 , 70 (1970).

${ }^{11} \mathrm{M}$. Cardona, in Proceedings of the Conference on Electronic Density of States, Gaithersburg, Md., 1969 (to be published). 